\title{
Time-Resolved Ultrafast All-Optical Switching in Directional Couplers with Second-Order Nonlinearity
}

\author{
Alexander S. Solntsev ${ }^{1}$, Dragomir N. Neshev ${ }^{1}$, and Roland Schiek ${ }^{2}$ \\ ${ }^{1}$ Nonlinear Physics Centre, Research School of Physics and Eng., Australian National University, Canberra, Australia \\ ${ }^{2}$ University of Applied Sciences Regensburg, Prüfeninger Strasse 58, 93049 Regensburg, Germany
}

Nonlinear directional couplers (NLDC) allow for ultrafast all-optical switching. To date, various types of NLDCs have been studied, predominantly based on the Kerr [1] or cascaded quadratic nonlinearity [2] in secondharmonic generation (SHG). In the later case the switching can occur at lower powers because of a propagatingwave resonance effect. While a number of experiments to characterise the pulse propagation in Kerr-type couplers exist, till now the temporal behavior of short pulses in the NLDC with quadratic nonlinearity has never been studied experimentally. Several important questions such as the reason for incomplete switching and possible pulse compression factors remain unanswered. In this work we experimentally measure the pulse reshaping in a NLDC with second-order nonlinearity and show that pulse compression, break-up and back-switching play an important role in the switching process.

In our experiments we use $8 \mathrm{ps}$, close to transform-limited pulses at a central wavelength of $1550.6 \mathrm{~nm}$. The NLDC is formed in a periodically poled lithium niobate (PPLN) crystal with two closely-spaced waveguides formed by Ti indiffusion [Fig. 1(a)]. Due to linear coupling between the waveguides at low input powers most of the energy is transferred from the input bar arm to the cross arm. When the input power increases, however, the coupler redirects the fundamental wave (FW) output to the bar arm due to a nonlinear detuning of the coupling by phase shifts induced due to the cascaded quadratic nonlinearity. For strong nonlinear response the phasematching was temperature tuned to a few $\mathrm{nm}$ above SHG phase-matching. Switching was characterized for positive nonlinear phase shifts in the low-FW-depletion regime. For temporal and spectral measurements we use a SHGbased frequency-resolved optical gating system. Fig. 1(b) demonstrates that the pulse in the cross arm suffers break-up at the switching input peak power. At higher powers, the center of the pulse is transferred to the bar channel, while the lower-power pulse wings remain in the cross channel. This behavior leads to pulse compression in both channels and can be understood such that the wings of the FW pulse do not generate enough nonlinear phase shift. The role of the SH is illustrated by the result that both sub-pulses in the cross channel are not equal in power. This is connected to a significant difference of the group velocities of FW and SH. With an increase of the input power above the switching point, the energy in both sub-pulses in the cross arm retains at the same ratio. Interestingly, in the bar arm [Fig. 1(c)] for an input peak power around three times the switching power, one can notice that the process of back-switching to the cross arm begins. The spectra of the pulses in both arms [Fig. 1(d,e)] are significantly broadened by self-phase modulation due to cascading, in agreement with the spectral shapes observed in single PPLN waveguides under similar conditions [3].
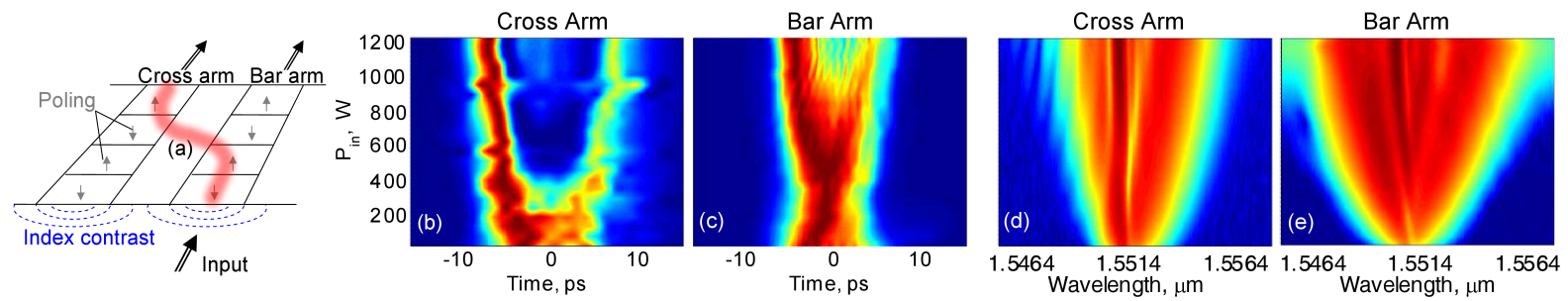

Fig. 1: (a) Scheme of the studied NLDC. (b, c) Pulse shapes in linear scale and (d, e) spectra in logarithmic scale for cross and bar arms accordingly in respect to input peak power.

The observed results show that the quadratic NLDC exhibit four orders of magnitude lower switching power than Kerr-based couplers, however their temporal dynamics is different because of the group-velocity mismatch between SH and FW and due to FW depletion and SHG.

\section{References}

[1] A. Villeneuve, C. C. Yang, P. G. J. Wigley, G. I. Stegeman, J. S. Aitchison, and C. N. Ironside, "Ultrafast all-optical switching in semiconductor nonlinear directional couplers at half the band gap," Appl. Phys. Lett. 61, 147 (1992).

[2] R. Schiek, L. Friedrich, H. Fang, G. I. Stegeman, K. R. Parameswaran, M. H. Chou, and M. M. Fejer, "Nonlinear directional coupler in periodically poled lithium niobate," Opt. Lett. 24, 1617 (1999).

[3] Z. Zheng, A. M. Weiner, K. R. Parameswaran, M.-H. Chou, and M. M. Fejer, "Femtosecond second-harmonic generation in periodically poled lithium niobate waveguides with simultaneous strong pump depletion and group-velocity walk-off," J. Opt. Soc. Am. B 19, 839 (2002). 